\title{
Sepse Brasil: Estudo Epidemiológico da Sepse em Unidades de Terapia Intensiva Brasileiras*
}

\author{
An Epidemiological Study of Sepsis in Intensive \\ Care Units. Sepsis Brazil Study
}

\begin{abstract}
João Andrade L. Sales Júnior', Cid Marcos David², Rodrigo Hatum³, Paulo César S. P. Souza ${ }^{4}$, André Japiassú ${ }^{5}$, Cleovaldo T. S. Pinheiro ${ }^{6}$, Gilberto Friedman ${ }^{7}$, Odin Barbosa da Silva ${ }^{8}$, Mariza D'Agostino Dias ${ }^{9}$, Edwin Koterba ${ }^{10}$, Fernando Suparregui Dias ${ }^{11}$, Cláudio Piras ${ }^{12}$, Grupo de Estudo de Sepse do Fundo AMIB ${ }^{13}$, Ronir Raggio Luiz ${ }^{14}$
\end{abstract}

\section{RESUMO}

JUSTIFICATIVA E OBJETIVOS: A sepse representa a principal causa de morte nas UTI em todo o mundo. Muitos estudos têm demonstrado um aumento da incidência ao longo do tempo e apenas uma leve redução na mortalidade.

MÉTODO: Foi realizado um estudo prospectivo em 65 hospitais de todas as regiões do Brasil. Os pacientes que foram admitidos com sepse ou que desenvolveram sepse no mês de setembro de 2003 foram incluídos. O acompanhamento foi feito até o $28^{\circ}$ dia de internação

1. Médico intensivista do Hospital de Clínicas de Niterói e do Instituto Nacional de Traumato-Ortopedia, Rio de Janeiro

2. Coordenador da UTI do Hospital Universitário Clementino Fraga Filho (HUCFF), Universidade Federal do Rio de Janeiro e da PósGraduação em Medicina Intensiva

3. Médico intensivista do Hospital São Lucas, Rio de Janeiro

4. Coordenador da UTI do Hospital de Clínicas de Niterói

5. Médico intensivista do Hospital Quinta Dor e HUCFF

6. Coordenador da UTI do Hospital de Clínicas de Porto Alegre

7. Coordenador da UTI do Complexo Hospitalar Santa Casa Porto Alegre-Rio Grande do Sul

8. Coordenador da UTI do Hospital Santa Joana, Recife

9. Coordenadora da UTI do Hospital Nove de Julho, São Paulo

10. Coordenador do H. São Camilo- São Paulo

11. Coordenador da UTI do Hospital São Lucas da PUC, Rio Grande do Sul

12. Coordenador da UTI do H. São Lucas. Vitória, ES

13. Grupo participante do Estudo Sepse Brasil

14. Prof. assistente de bioestatística da Universidade Federal do Rio de Janeiro.

*Recebido pela Universidade Federal do Rio de Janeiro

Apresentado em 16 de janeiro de 2006

Aceito para publicação em 03 de março de 2006

Endereço para correspondência

Dr. João Andrade L. Sales

Praça Antonio Callado, 215/1701

22793-081 Rio de Janeiro, RJ

Fone: (21) 2429-5537; Fax: (21) 2729-5537

Email: jandradesales@hotmail.com e/ou até a alta da UTI. O diagnóstico seguiu os critérios clássicos propostos na convenção de 1991. Foram avaliados dados demográficos, escore APACHE II, escore SOFA, mortalidade, fonte de infecção, microbiologia, comorbidades, tempo de internação, uso de ventilação mecânica, cateter de Swan-Ganz, vasopressores e hemotransfusão.

RESULTADOS: Setenta e cinco unidades de terapia intensiva de todas as regiões do Brasil participaram do estudo. Foram identificados 3128 pacientes e 521 (16,7\%) foram diagnosticados como tendo o diagnóstico de sepse, sepse grave ou choque séptico. A idade média foi de 61,7 (IQR 39-79), 293 (55,7\%) corresponderam ao sexo masculino, e a mortalidade global em 28 dias foi de 46,6\%. O escore APACHE II médio foi de 20 e o escore SOFA no D1 foi de 7 (IQR 4-10). O escore SOFA no grupo dos não-sobreviventes foi maior no D1 (8, IQR 5-11), e aumentou no D3 (9, IQR 6-12). A mortalidade na sepse, sepse grave e choque séptico foi de $16,7 \%, 34,4 \%$ e $65,3 \%$, respectivamente. O tempo médio de internação foi de 15 dias (IQR 5-22). As duas principais fontes de infecção foram o trato respiratório (69\%) e o abdômen $(23,1 \%)$. Os bacilos gram-negativos foram mais prevalentes $(40,1 \%)$. Os cocos gram-positivos foram identificados em $32,8 \%$ e as infecções fúngicas em $5 \%$. A ventilação mecânica ocorreu em $82,1 \%$ dos casos, uso de cateter de Swan-Ganz em 18,8\%, vasopressores em $66,2 \%$ e hemotransfusão em $44,7 \%$ dos casos.

CONCLUSÕES: O estudo evidenciou uma elevada mortalidade da sepse nas UTI em nosso país. A mortalidade no choque séptico é uma das mais elevadas no mundo. Nossos pacientes são mais graves e com tempo de internação maior. O momento é muito propício a uma reflexão ainda maior sobre esta doença que é a principal causa de morte nas UTI, haja vista o elevado impacto econômico e social. Precisa-se cada vez mais desenvolver Campanhas de Sobrevivência na Sepse e fazer uso 
racional, baseado em evidências, dos recursos por ora disponíveis e da forma mais precoce possível.

Unitermos: choque séptico, epidemiologia, sepse, sepse grave

\section{SUMMARY}

BACKGROUND AND OBJECTIVES: Sepsis represents the major cause of death in the ICUs all over the world. Many studies have shown an increasing incidence over time and only a slight reduce in mortality. Many new treatment strategies are arising and we should define the incidence and features of sepsis in Brazil.

METHODS: Prospective cohort study in sixty-five hospitals all over Brazil. The patients who were admitted or who developed sepsis during the month of September, 2003 were enrolled. They were followed until the $28^{\text {th }}$ day and/or until their discharge. The diagnoses were made in accordance to the criteria proposed by ACCP/SCCM. It were evaluated demographic features, APACHE II score, SOFA (Sepsis-related Organ Failure Assessment) score, mortality, sources of infections, microbiology, morbidities and length of stay (LOS).

RESULTS: Seventy-five ICUs from all regions of Brazil took part in the study. 3128 patients were identified and $521(16.7 \%)$ filled the criteria of sepsis, severe sepsis or septic shock. Mean age was 61.7 (IQR 39-79), 293 $(55.7 \%)$ were males, and the overall 28 -day mortality rate was $46.6 \%$. Average APACHE II score was 20 and SOFA score on the first day was 7 (IQR 4-10). SOFA score in the mortality group was higher on day 1 (8, IQR 5-11), and had increased on day 3 (9, IQR 6-12). The mortality rate for sepsis, severe sepsis and septic shock was $16.7 \%, 34.4 \%$ and $65.3 \%$, respectively. The average LOS was 15 days (IQR 5-22). The two main sources of infection were the respiratory tract $(69 \%)$ and the abdomen one (23.1\%). Gram-negative bacilli were more prevalent $(40.1 \%)$. Gram-positive cocci were identified in $32.8 \%$ and fungi infections in $5 \%$. Mechanical ventilation was observed in $82.1 \%$ of the patients, Swan-Ganz catheter in $18.8 \%$, vasopressors in $66.2 \%$ and hemotransfusion in $44.7 \%$.

CONCLUSIONS: It was observed a high mortality of sepsis in the ICUs in Brazil. The high frequency of septic shock demonstrated a group at high risk of death. In order to have a better use of the resources and reduce in mortality during the next 5 years, it is very important to identify our specific features related to this syndrome.

Key Words: Epidemiology; sepsis; severe sepsis; septic shock.

\section{INTRODUÇÃO}

A sepse é a principal causa de morte nas unidades de terapia intensiva (UTI) e está entre as principais causas de morte nos EUA ${ }^{1}$. Em torno de $2 \%$ a $11 \%$ das internações hospitalares e nas UTI são por esta doença ${ }^{2}$. A mortalidade varia na maioria dos estudos entre $20 \%$ e $80 \%^{3}$. Martin e col. demonstraram que houve aumento da incidência no período de 1979 (82,7/100,000 população) a 2000 (240,4/100,000 população), em torno de $8,7 \%$ ao ano. A mortalidade reduziu de $27,8 \%$ nos primeiros anos para $17,9 \%$ nos últimos 05 anos de avaliação ${ }^{1}$. Annane e col. publicaram tendência semelhante na incidência e mortalidade quando avaliaram pacientes com choque séptico em um período de oito anos. As internações na UTI elevaram-se de 7 (em 1993) para 9,7 (em 2000) a cada 100 internações. A mortalidade reduziu de $62,1 \%$ (em 1993) para 55,9\% (em 2000)4. Angus e col., estimaram uma incidência anual nos EUA de 751.000 casos (3 casos/1.000 população) e evolução para óbito em 215.000 casos $(28,6 \%)^{5}$.

No início do século XXI ainda busca-se uma compreensão melhor da doença. Não se pode negar avanços em relação ao diagnóstico mais precoce, rastreamento microbiano mais eficaz que possibilita o rápido início do tratamento, o uso mais otimizado das variáveis hemodinâmicas e das técnicas de suporte orgânico. Avançase também para um controle metabólico mais eficaz, compreendendo a importância do controle glicêmico ${ }^{6}$, diagnosticando e tratando mais freqüentemente a insuficiência adrenal relativa ${ }^{7}$. A teoria, a utilização da ventilação mecânica e terapia nutricional estão igualmente mais bem administrados ao paciente grave. Surgem freqüentemente novas medicações que visam interferir na cascata inflamatória e da coagulação, todavia sem uma resposta convincente na maior parte das vezes. A proteína $\mathrm{C}$ ativada recombinante humana mostrou redução na mortalidade e seus resultados são bastante relevantes e confirmam a importância da interferência nos mecanismos inflamatórios e da coagulação ${ }^{8,9}$. Nos anos que se seguirão com o desenvolvimento cada vez maior da genômica, da proteômica e da bioengenharia certamente ocorrerão avanços significativos na compreensão da fisiopatologia e na instituição de terapêuticas cada vez mais eficazes. Os estudos em genética parecem apontar para um tratamento molecular, individualização e interferência direta em mecanismos celulares como a apoptose e no microcosmo endotelial. O presente estudo visou compreender melhor a epi- 
demiologia da sepse no Brasil e conseqüentemente facilitar no futuro a implementação de projetos educacionais, de novos avanços diagnósticos e terapêuticos e recursos materiais. Em um país com dimensão continental, é muito importante se conhecer os dados epidemiológicos globais e regionais. Este é o primeiro estudo de âmbito nacional nesta questão.

\section{MÉTODO}

Foi realizado um estudo de coorte, prospectivo, multicêntrico, em 65 hospitais de todas as regiões do Brasil. O estudo foi coordenado pelo Fundo Brasileiro de Educação e Pesquisa da AMIB (Fundo AMIB - Associação de Medicina Intensiva Brasileira) e pela Pós-graduação da Universidade Federal do Rio de Janeiro (Clínica Médica- área Terapia Intensiva). O protocolo de estudo foi aprovado pelo Comitê de Ética em Pesquisa. Foram convidadas unidades de terapia intensiva (UTI) de todo o Brasil. Os hospitais participantes receberam um caderno para preenchimento dos dados e com as instruções pertinentes.

O período de inclusão foi de 30 dias (1 a 30 de setembro de 2003), e cada paciente acompanhado por 28 dias ou até sua alta da UTI ou óbito. Foram incluídos todos os pacientes internados com sepse ou que desenvolveram sepse no período do estudo. O diagnóstico de sepse seguiu as orientações clássicas da conferência de consenso de $1991^{10}$. Foram excluídos os pacientes menores de 18 anos, de re-internação no mesmo período do estudo, de perda de acompanhamento por transferência e de internação na UTI por tempo inferior a 24 horas.

Foi utilizado como índice prognóstico o escore APACHE II (Acute Physiologic and Chronic Health Evaluation) e o escore SOFA (Sepsis-Related Organ Failure Assessment) ${ }^{11}$ para acompanhamento das disfunções orgânicas. O escore SOFA foi medido no $1^{\circ}$ dia (D1), $3^{\circ} \mathrm{dia}(\mathrm{D} 3), 5^{\circ} \mathrm{dia}(\mathrm{D} 5), 7^{\circ} \mathrm{dia}$ (D7) e $14^{\circ} \mathrm{dia}$ (D14) após a inclusão do paciente no estudo. O escore APACHE II utilizado foi o da internação na UTI. Foram ainda anotados os dados demográficos, a mortalidade em 28 dias, comorbidades, fonte de infecção, microbiologia e tempo de internação. O diagnóstico de infecção foi feito de acordo com os critérios de cada hospital. O diagnóstico do estágio evolutivo da sepse foi feito considerando-se o estágio mais grave nos quatro primeiros dias de acompanhamento. Avaliou-se ainda se o paciente foi submetido a ventilação mecânica, hemotransfusão, uso de vasopressores e uso de cateter de
Swan-Ganz.

Para comparação dos dados quantitativos entre dois grupos foi utilizado o teste $t$ de Student para amostras independentes ou teste não-paramétrico de MannWhitney. Para comparação de dados qualitativos foi aplicado o teste Qui-quadrado $\left(\chi^{2}\right)$ ou teste Exato de Fisher. O coeficiente de correlação de Pearson avaliou o grau de associação entre variáveis numéricas. $\mathrm{O}$ critério de determinação significativa adotado foi de $5 \%$. A análise estatística foi processada pelo software estatístico $S A S \otimes$ System.

\section{RESULTADOS}

Foram convidadas $140 \mathrm{UTI}$, sendo que das $81(57,8 \%)$ que participaram do estudo, seis UTI foram excluídas por apresentarem dados incompletos. Os dados foram então analisados em 75 UTI, correspondendo a 65 hospitais de todas as regiões do Brasil (Figura 1). As regiões sul e sudeste tiveram a maior participação, $73,8 \%$

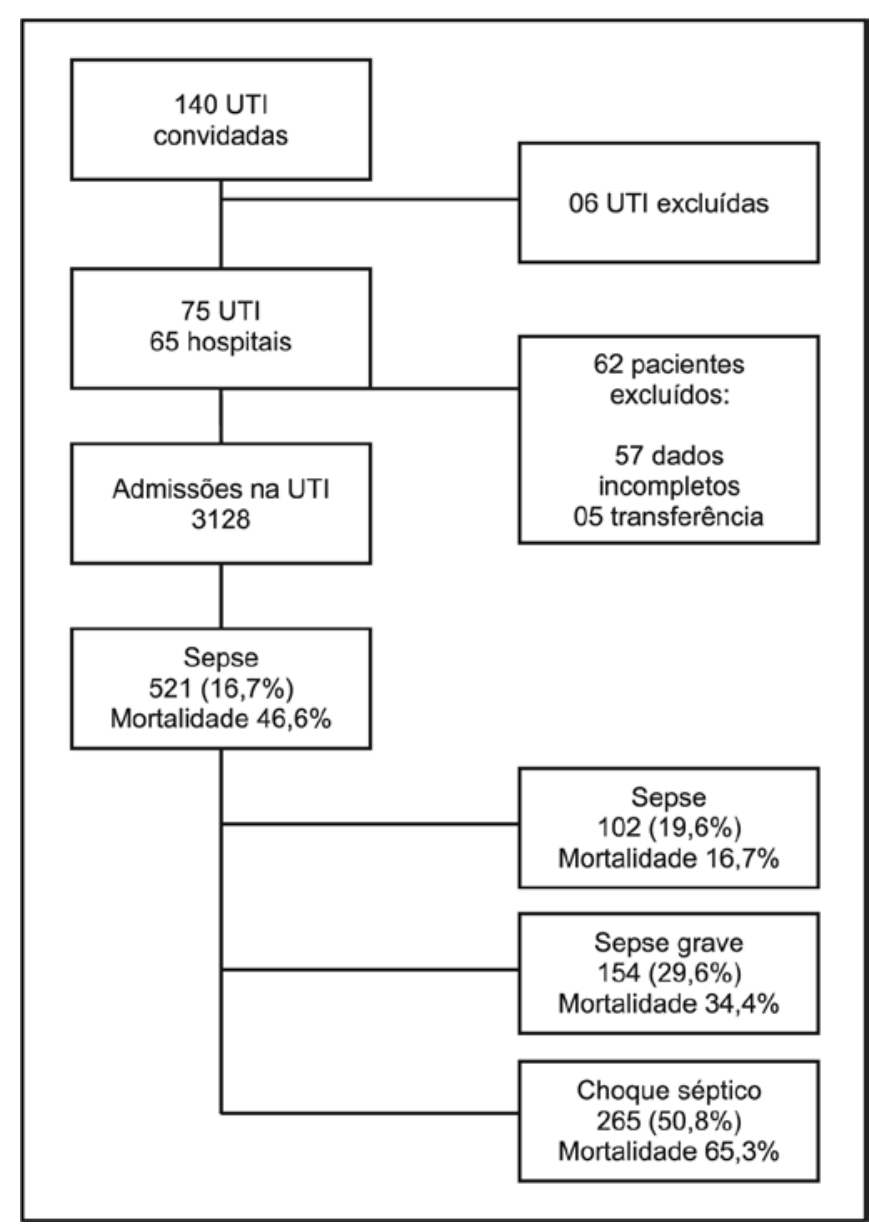

Figura 1 - Diagrama com Pacientes, UTI e Hospitais Incluídos, além da Mortalidade Global e nos Subgrupos Sepse, Sepse Grave e Choque Séptico. 
Tabela 1 - Distribuição das UTI Participantes e Dados Globais e Regionais.

\begin{tabular}{l|c|c|c|c}
\hline & Total & Região Sudeste & Região Sul & Regiões:Nordeste, Norte e Centro-oeste \\
\hline Hospitais (n e \%) & 65 & $40(61,5)$ & $8(12,3)$ & $17(26,1)$ \\
H. Privado (n) & 42 & 26 & 3 & 13 \\
H. Público (n) & 23 & 14 & 5 & 4 \\
Pacientes (n e \%) & 521 & $344(66)$ & $103(19,8)$ & $74(14,2)$ \\
Idade média (IQ) & $61,7(39-79)$ & $63,9(53-78)$ & $56,1(42-70)$ & $58,3(38,5-75,5)$ \\
Sexo M/F (\%) & $55,7 / 44,3$ & $56,3 / 43,7$ & $52,4 / 47,6$ & $58,6 / 41,4$ \\
APACHE II médio (IQ) & $20(15-25)$ & $19(14-23)$ & $23,5(18-29)$ & $22,2(16,8-27)$ \\
SOFA D1 médio (IQ) & $7(4-10)$ & $6,7(4-9)$ & $7,9(5-10)$ & $7,3(5-10)$ \\
Tempo de internação médio (IQ) & $15(5-22)$ & $16,5(6-22)$ & $12,2(5-18)$ & $16(7-23,8)$ \\
Mortalidade global (\%)* & 46,6 & 43 & 52,4 & 55,4 \\
Sepse (\%) ** & 19,6 & 21 & 21,4 & 10 \\
Sepse grave (\%)* & 29,6 & 27,6 & 29,1 & 40 \\
Choque séptico (\%)** & 50,8 & 51,4 & 49,5 & 50 \\
\hline
\end{tabular}

*A mortalidade apresentada em cada região refere-se, de fato, ao percentual local.

${ }^{\star *} \mathrm{~A}$ incidência de sepse, sepse grave e choque séptico refere-se ao percentual em relação ao total de pacientes em cada região.

dos hospitais e $85,8 \%$ dos pacientes do estudo (Tabela 1). A média de leitos das UTI foi de 12,5 (4-39). A maior parte das UTI foi classificada como UTI geral $(86,2 \%)$, as demais se distribuíram entre cardiológica $(6,2 \%)$, pós-operatório $(3,1 \%)$, trauma $(1,5 \%)$ e mistas. Houve uma predominância de hospitais privados $(64,6 \%)$, todavia, a mortalidade, foi um pouco maior nos hospitais da rede pública $(48,1 \%$ versus $45,6 \% ; p=0,577)$. Foram admitidos no período do estudo 3128 pacientes e $521(16,7 \%)$ apresentaram sepse (Figura 1). A sepse, sepse grave e o choque séptico ocorreram em 19,6\%, $29,6 \%$ e $50,8 \%$ dos casos, respectivamente. As internações foram predominantemente de natureza clínica $(67,2 \%)$, seguindo-se os pós-operatórios de emergência $(24,4 \%)$ e os eletivos $(8,4 \%)$. Predominou o sexo masculino $(55,7 \%)$ e a raça branca $(80,1 \%)$. A idade média foi de 61,7 anos (IQ 39-79). O escore APACHE II médio foi de 20 (IQ 15-25) (Tabela 2). O escore SOFA médio no primeiro dia (D1) foi de 7 (IQ 4-10) e, a partir do terceiro dia (D3), elevou-se nos não sobreviventes ( $p<0,001$ : figura 3). A mortalidade global no grupo dos sépticos foi de 46,6\% (IC 95\% 42,3\%-51\%). A mortalidade nos subgrupos sepse, sepse grave e choque séptico foram de $16,7 \%, 34,4 \%$ e $65,3 \%$, respectivamente (Tabela 3 e Figura 2). A mortalidade em 28 dias foi maior no sexo masculino $(48,5 \%$ e $44,3 \%, p=0,597)$ e nos não-brancos $(50 \%$ e $45,5 \%, p=0,417)$. A mortalidade também foi maior nos pacientes submetidos a ventilação mecânica $(54,1 \%$ versus $12,9 \%, p<0,001)$, hemotransfusão $(50,6 \%$ versus $43,6 \%, p=0,101)$, que usaram cateter de Swan-Ganz (54,1\% versus $44,9 \%$, $\mathrm{p}=0,101)$ e nos que necessitaram de vasopressores $(59,4 \%$ versus $21,8 \%, p<0,001)$ (Tabela 2$)$. Os prin-
Tabela 2 - Dados Demográficos e Gerais

\begin{tabular}{l|c}
\hline \multicolumn{1}{c|}{ Variáveis } & Geral \\
\hline Número / Porcentagem & $521(16,7)$ \\
Idade média (IQ) & $61,7(39-79)$ \\
Sexo M/F (\%) & $55,7 / 44,3$ \\
Raça branca (\%) & 80,1 \\
APACHE II médio (IQ) & $20(15-25)$ \\
SOFA D1 médio (IQ) & $7(4-10)$ \\
SOFA D3 médio (IQ) & $7,3(4-10)$ \\
Internação clínica (\%) & 67,2 \\
Internação pós-op. Eletiva (\%) & 8,4 \\
Internação pós-op. Emergência (\%) & 24,4 \\
Infecção respiratória (\%) & 69 \\
Infecção abdominal (\%) & 23,1 \\
Infecção urinária (\%) & 16 \\
Sepse (n e \%) & $102(19,6)$ \\
Sepse grave (n e \%) & $154(29,6)$ \\
Choque séptico (n e \%) & $265(50,8)$ \\
Mortalidade global (n e \%) & $46,6 \%$ \\
Tempo de internação médio (IQ) & $15(5-22)$ \\
Ventilação mecânica (n e \%) & $427(82,1 \%)$ \\
Cateter Swan-Ganz (n e \%) & $98(18,8 \%)$ \\
Vasopressores (n e \%) & $345(66,2 \%)$ \\
Hemotransfusão (n e \%) & $233(44,7 \%)$ \\
\hline
\end{tabular}

cipais locais de infecção (Figura 4) foram o pulmonar (69\%), seguindo-se o abdominal $(23,1 \%)$ e o urinário (16\%). A mortalidade, entretanto, foi maior nos casos de bacteremia ( $n=18 ; 55,6 \%$ ), fonte abdominal $(n=$ $62 ; 51,7 \%)$ e, em seguida, pulmonar ( $n=171 ; 47,8 \%)$. A Pseudomonas aeruginosa e o Staphyfilococos aureus meticilino-resistente predominaram (Figura 6). O diagnóstico de sepse fúngica ocorreu em $5,2 \%(n=27)$ dos casos. O tempo de internação médio foi de 15 dias (IQ 
Tabela 3 - Avaliação entre os Grupos Sepse, Sepse Grave e Choque Séptico.

\begin{tabular}{|c|c|c|c|c|}
\hline & Sepse & Sepse Grave & Choque Séptico & p-valor \\
\hline$N(\%)$ & $102(19,6 \%)$ & $154(29,6 \%)$ & $265(50,8 \%)$ & $<0,001$ \\
\hline Mortalidade (\%) & 16,7 & 34,4 & 65,3 & $<0,001$ \\
\hline APACHE II médio (IQ) & $16,8(11-22)$ & $19,4(15-23)$ & $22,2(17-27)$ & $<0,001$ \\
\hline SOFA médio D1 (IQ) & $3,8(2-6)$ & $6,2(4-8)$ & $8,8(6-11)$ & $<0,001$ \\
\hline SOFA médio D3 (IQ) & $3,4(1-5)$ & $6,2(4-8)$ & $9,5(7-12)$ & $<0,001$ \\
\hline Tempo internação UTI, média (IQ) & $13,5(6-16)$ & $16,8(7-23)$ & $15,6(5-22)$ & $=0,015$ \\
\hline
\end{tabular}

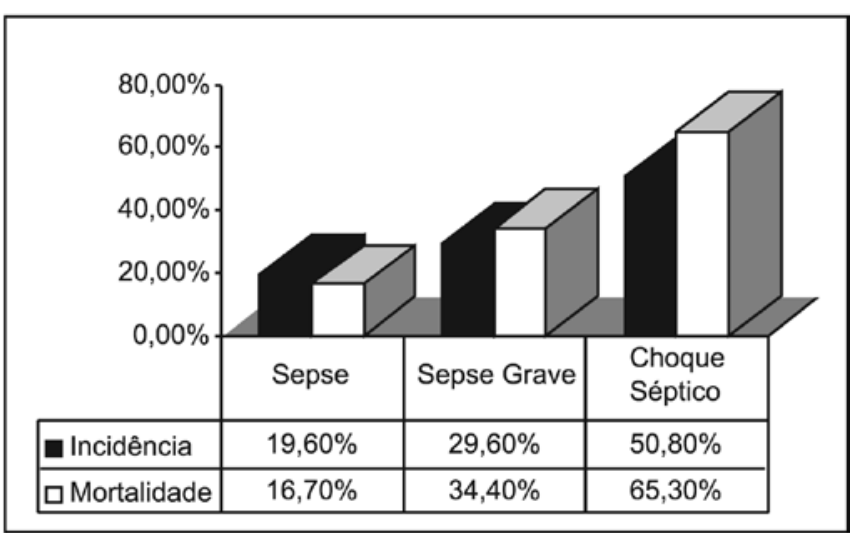

Figura 2 - Incidência e Mortalidade na Sepse, Sepse Grave e Choque Séptico

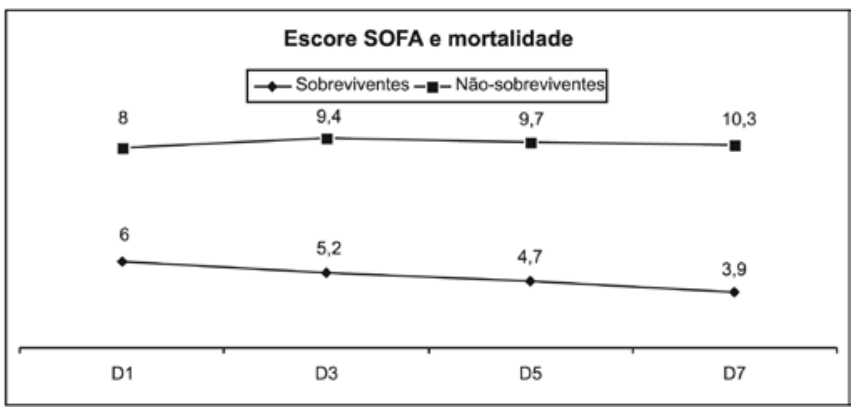

Figura 3 - Escore SOFA nos Sobreviventes e Não Sobreviventes.

Em todos os dias assinalados houve estatística significância $(p<0,001)$. Valor médio em cada dia assinalado.

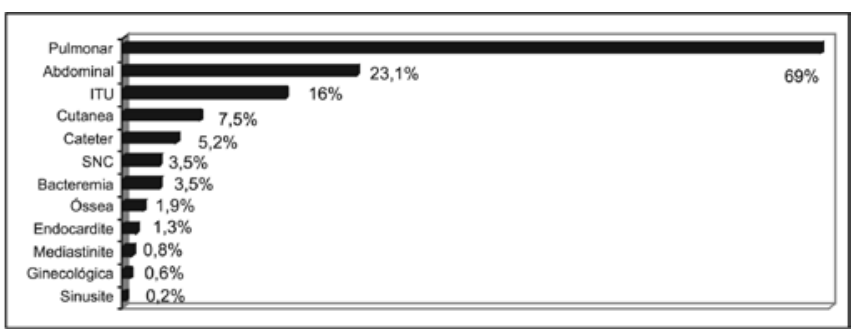

Figura 4 - Fontes de Infecção

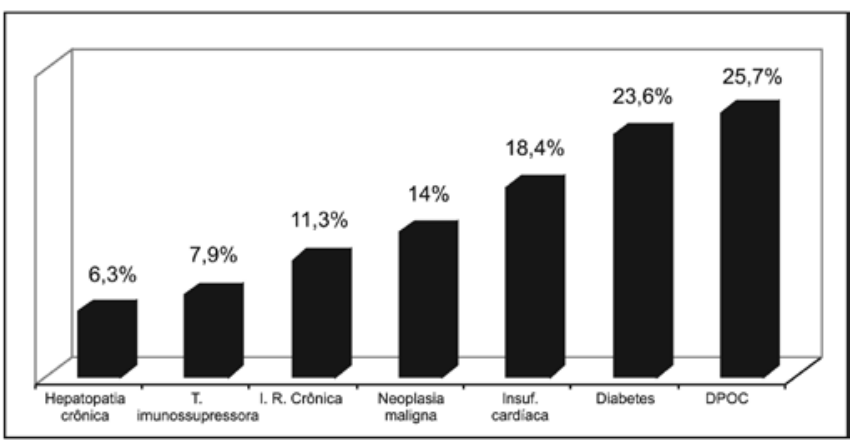

Figura 5 - Comorbidades

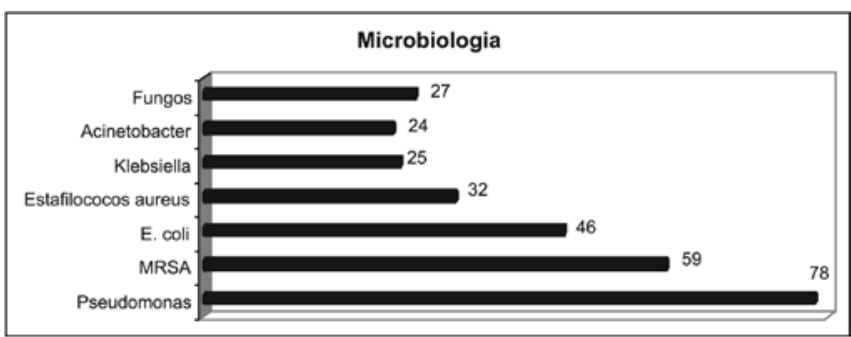

Figura 6 - Principais Microorganismos Identificados.

Os números referem-se ao número de culturas positivas para cada patógeno. Os bacilos gram-negativos representaram 40,1\% , cocos gram-positivos $32,8 \%$ e fungos $5 \%$.

MRSA: Stafhylococos aureus meticilino resistente

5 -22). Este período foi um pouco mais prolongado nos pacientes com sepse grave ou choque séptico (16,3;IQ $7-23,3)$. As principais comorbidades se referiram ao aparelho cardiovascular e respiratório. Destacam-se a doença pulmonar obstrutiva crônica (DPOC) $(25,7 \%$ dos casos), a insuficiência cardíaca ( $18,4 \%$ dos casos) e a diabete melito $(23,6 \%$ dos casos) (Figura 5). É relevante ainda assinalar que $14 \%$ dos pacientes apresentavam o diagnóstico associado de neoplasia maligna e $8 \%$ do total de pacientes sépticos estavam sob terapia imunossupressora. A insuficiência renal crônica (IRC) fora identificada em $11,3 \%$ dos casos. Quando se avaliou a mortalidade associada a cada comorbidade, ela foi mais elevada nos hepatopatas crônicos $(66,7 \%)$, 
seguindo-se os pacientes sob terapia imunossupressora $(58,5 \%)$, os portadores de IRC $(57,6 \%)$ e os portadores de DPOC (53,7\%). A mortalidade relacionou-se com o número de comorbidades, atingindo $56 \%$ nos portadores de três doenças associadas $(p=0,036)$

\section{DISCUSSÃO}

A sepse permanece como um grande desafio em todo o mundo e não é diferente em nosso país. O estudo congregou um número significativo de UTI em todo o país e com distribuição proporcional. A mortalidade global dos pacientes sépticos foi de 46,6\% (243 pacientes). Quando se avaliou os pacientes com choque séptico, a mortalidade encontrada foi de $65,3 \%$ e na sepse grave de $34,4 \%$. A mortalidade na sepse grave é semelhante à encontrada em estudo em 206 UTI na França $(35 \%)^{12}$ e inferior a encontrada no estudo do Reino Unido $(46 \%)^{13}$. A mortalidade no choque séptico foi bastante elevada (65\%), superior ao do estudo de Annane e col. (62,1\% em 1993 e 55,9\% em 2000). Em uma revisão da mortalidade no choque séptico a mortalidade global encontrada foi de $49,7 \%$, com variação em torno de $40 \%$ a $80 \%{ }^{3}$. O estudo SOAP, realizado em 198 UTI Européias, demonstrou uma mortalidade na sepse grave de $32,2 \%$ e no choque séptico de $54,1 \%{ }^{14}$. Eliézer e col. mostraram recentemente no estudo BASES, mortalidade na sepse grave e choque séptico de $46,9 \%$ e $52,2 \%$, respectivamente ${ }^{15}$. Este estudo, também brasileiro, apresenta dados epidemiológicos semelhantes ao presente estudo, incluindo idade, predominância do sexo masculino, valores do escore APACHE II e do escore SOFA e predominância do sítio pulmonar. A idade média observada (61,7 anos) e a predominância do sexo masculino é uma constante em todos os estudos. Há uma tendência ao aumento da faixa etária e a uma diferença que pouco mudou em relação aos gêneros. $O$ aumento da expectativa de vida, os avanços tecnológicos que permitem prolongar e salvar mais vidas, contribuem para esta constatação. O escore APACHE II relacionou-se bem com mortalidade e gravidade $(p<0.05)$. O escore SOFA apresentou notável relação com evolução para óbito ou não. Quando se observa a evolução das disfunções orgânicas, tem-se que o terceiro dia de evolução da sepse grave e choque séptico parece ser um marco que discriminará uma tendência para óbito ou não $(p<0.001)$ (figura 3) (D3). Esta observação é mais um dado a reforçar a campanha mundial de sobrevivência na sepse (Survival Sepsis Campaign), na qual as sociedades médicas em todo o mundo apregoam abordagens rápidas, precoces e protocoladas das ações terapêuticas ${ }^{16}$. Elas planejam uma redução na mortalidade da sepse de $25 \%$ até 2010. O estudo Sepse Brasil evidenciou um número de pacientes com sepse grave e choque séptico maior que os relatados nas publicações européias e norte-americanas. Nestes outros estudos o número de pacientes com sepse sem classificação como graves é maior. Vale ressaltar que não foram contados episódios de sepse, nem qualquer paciente foi incluído duas vezes no estudo. Se for considerado o contexto da UTI, é de se esperar que se internem mais pacientes com sinais de hipoperfusão do que apenas com os sinais algo inespecíficos de SIRS e um foco de infecção ${ }^{17,18}$. O momento de classificar um paciente séptico é algo também muito controverso. Um estudo exclusivo de sepse grave terá certamente avaliado resultados finais de um percentual razoável de pacientes que evoluíram com choque séptico. Considerando a sepse como uma doença de evolução dinâmica, e que se define em sua gravidade, habitualmente, nos primeiros dias, considera-se razoável fazer a classificação ao final do quarto dia do diagnóstico de sepse. Frausto e col. demonstraram em 2527 pacientes com SIRS a evolução para sepse, sepse grave e choque séptico em $26 \%$, $18 \%$ e $4 \%$, respectivamente. Neste mesmo estudo o tempo médio de evolução de sepse para sepse grave foi de $01 \mathrm{dia}^{18}$. Não se têm as informações do tempo de espera nas salas de emergência ou enfermarias para a internação na UTI, entretanto sabe-se que se trata de um importante problema em nosso país. Em muitos de nossos pacientes pode ter havido atraso na ida para a UTI e, conseqüentemente, pode ter aumentado o número de casos de sepse grave e choque séptico. Nosso tempo de internação também está maior (média de $15,5 \pm 11$ dias) $1,12,13,15,18-20$. O estudo BASES, mostra um tempo de internação na UTI baixo (1 a 6 dias), todavia congregou um número menor de hospitais. O número elevado de pacientes sépticos por pneumonia está em

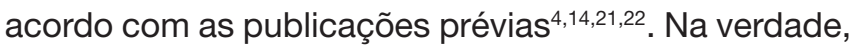
cada vez mais o sítio respiratório tem sido implicado na fonte do processo infeccioso, o que é compatível com um número cada vez maior de pacientes sob ventilação mecânica e com internação prolongada nas unidades de terapia intensiva. Lidamos, de fato, no presente estudo com uma população de pacientes muito graves, haja vista a alta incidência de pacientes que usaram a ventilação mecânica (82\%), vasopressores $(66,2 \%)$, hemotransfusão $(44,7 \%)$ e cateter de SwanGanz (18,8\%). Em nosso meio predominam as bacté- 
rias gram-negativas, com a Pseudomonas aeruginosa sendo seu principal representante. Foram muito preocupantes os achados de patógenos multiresistentes: os Staphylococos aureus meticilino resistentes (MRSA) foram o segundo microrganismo mais encontrado. Vincent e col. demonstraram uma incidência elevada de Staphylococos aureus meticilino resistentes (MRSA), Pseudomonas species e fungos e com mortalidade elevada associada a eles ${ }^{14}$. As publicações nos principais periódicos de clínica médica e Medicina Intensiva alertam freqüentemente para este problema da crescente multiresistência em todos os continentes ${ }^{21,23-25}$. Nos EUA e em alguns países da Europa as bactérias gram-positivas já predominam, com destaque para os enterococos e estafilococos. Este último fato não ocorre até o momento no Brasil. Ainda no tocante a microbiologia há um aumento crescente das infecções fúngicas (5\% no presente estudo) e não somos exceção ${ }^{1,14}$. Chamam atenção a presença de comorbidades na população estudada, sobremaneira as ligadas aos aparelhos cardiovascular e respiratório. Este é o reflexo de estarmos lidando nas UTI de todo o país com uma população de idade cada vez mais avançada. $\mathrm{Na}$ análise das regiões, agruparam-se as regiões norte, nordeste e centro-oeste em face do número menor de pacientes (Tabela 2). A mortalidade foi um pouco maior neste grupo de regiões, e houve maior incidência de casos de sepse grave. A região sul apresentou uma mortalidade maior em relação a região sudeste, contudo lidou provavelmente com uma população mais grave se forem considerados os escores APACHE II e SOFA no $1^{\circ}$ dia (D1). Houve pouca diferença na idade e gênero, havendo uma tendência a uma população mais jovem e a uma equalização dos sexos na região sul. O estudo apresenta algumas limitações. A classificação da sepse no quarto dia (D4) não é um consenso. O acompanhamento somente até o $28^{\circ}$ dia (D28) certamente gera questionamentos quanto a mortalidade final da população estudada. O critério para diagnóstico das infecções baseou-se nos critérios de cada instituição, e não temos como avaliar cada critério regional utilizado. $\mathrm{O}$ fato de o estudo ter sido feito em somente um mês e, especificamente o mês de setembro, pode ter gerado viés, sobretudo em relação às infecções respiratórias. Entretanto, trata-se do primeiro grande estudo de âmbito nacional da sepse em UTI em países em desenvolvimento, e certamente representa um avanço na melhor compreensão do perfil nacional e na disseminação de conceitos relativos a esta doença.

$\mathrm{O}$ aumento da complexidade das UTI também pos- sui seus paradoxos: o aumento da invasibilidade e o uso indiscriminado dos novos antibióticos têm gerado complicações diversas, internações não raro muito prolongadas e com alto custo econômico e social. As UTI no Brasil precisam refletir o uso racional dos novos recursos e investir cada vez mais na educação continuada e nas discussões éticas de muitas questões na terapia intensiva.

\section{CONCLUSÃO}

O estudo evidenciou uma elevada mortalidade da sepse nas UTI em nosso país. A mortalidade no choque séptico é uma das mais elevadas no mundo. Nossos pacientes são mais graves e com tempo de internação maior. O momento é muito propício a uma reflexão ainda maior sobre esta doença que é a principal causa de morte nas UTI, haja vista o elevado impacto econômico e social. Precisamos nos engajar cada vez na Campanha de Sobrevivência na Sepse e fazer uso racional, baseado em evidências, dos recursos por ora disponíveis e da forma mais precoce possível.

\section{ABREVIAÇÕES:}

APACHE II - Acute Physiological and Chronic Health Evaluation II

DPOC - Doença Pulmonar Obstrutiva Crônica

IRC - Insuficiência Renal Crônica

LOS - Tempo de permanência

SOFA - Sepsis - Related Organ Failure Assessment

SIRS - Síndrome da Resposta Inflamatória Sistêmica

T.I. - Terapia imunossupressora

UTI / ICU - Unidade de Tratamento Intensivo

\section{PARTICIPANTES DO ESTUDO SEPSE BRASIL:}

Hospital Universitário - UFMS. Campo Grande-MS. Sérgio Félix Pinto;Hospital Madre Tereza. Belo Horizonte-MG. José Carlos F Versiani dos Anjos; Hospital São Lucas da PUC-RS. Porto Alegre-RGS. Fernando Suparregui Dias; Clínica Santa Marta. Rio de JaneiroRJ. Erika Ferraz de Gouvêa; H. São Vicente de Paulo. Rio de Janeiro-RJ. Guilherme Ribeiro Aguiar; Hospital UNIMED João Pessoa. João Pessoa -PB. Ciro Leite Mendes; Central Rio Médica. Rio de Janeiro-RJ. Eduardo Paranhos; Hospital Evangélico. Cachoeiro Itapemirim-ES. Marcellus Grilo e Hemerson Gama; Hospital Santa Helena. Goiânia-GO. Gleida Alves Borges; Hospital São Mateus. Fortaleza-CE. Joel Isidoro Costa; H. 
Santa Casa M. São João Del Rey. São João Del ReyMG. Jorge Paranhos; Hospital de Doenças Tropicais. Goiânia-GO. Arnaldo Zambrana Estrada; H. Clínicas de Niterói. Niterói-RJ. Paulo Cesar S. P. Souza; H. Quinta DOR. Rio de Janeiro-RJ. Andre Japiassú; H. Venerável Ordem Terceira da Penitência. Rio de Janeiro-RJ. Silvana W. Lopes de Lima; H. M. Lourenço Jorge. Rio de Janeiro-RJ. Deborah M. C. Haringer; H. Santa Cruz. Niterói-RJ. Aloysio Guimarães da Fonseca; H.Santa Izabel. Salvador-BA. Rosenbert Mamedio da Silva; H. U. Cassiano Antonio Moraes. Vitória-ES. Eliana Caser; H. São Lucas. Vitória-ES. Claudio Piras; H. de Base. São José do Rio Preto-SP. Suzana M. A. Lobo; H. Estadual Adão Pereira Nunes/EMCOR. Duque de Caxias-RJ. Antonio Ribeiro Pontes Neto; H. Naval Marcílio Dias. Rio de Janeiro-RJ. Celso Coelho; H. Clínicas de Nova Iguaçu. Nova Iguaçu-RJ. Mozart de Oliveira Netto; H. Amparo Feminino. Rio de Janeiro-RJ. Simone da S. Vinhas; $\mathrm{H}$. Porto Dias. Belém-PA. Romulo Nina; H. Emerg. e Trauma S. Humberto Lucena. João Pessoa-PB. Yuzeth Nóbrega; H. São Lucas. Rio de Janeiro-RJ. Marcos Knibel; H. Biocor. Nova Lima-MG. Maria Aparecida Braga; C. Saúde Santa Lúcia. Rio de Janeiro-RJ. Celina Acra; Associação Caridade Santa Casa do Rio Grande. Rio Grande-RGS. Luis Eduardo Correa Schein; H. Divina Providência. Porto Alegre-RGS. Rogério Fernandes; $\mathrm{H}$. São João Batista. Macaé-RJ. Joel Tavares Passos; $H$. Escola Padre Albino. Catanduva-SP. Jorge Luis Valiatti; H. Clínicas de Porto Alegre. Porto Alegre-RGS. Cleovaldo T. S. Pinheiro; H. Dorio Silva. Serra-ES. Francisca Alves Neto; H. Parque Belém. Porto Alegre-RGS. Jacqueline Froemming; H. Copa Dor. Rio de Janeiro-RJ. José Eduardo Couto de Castro; H. UNIMED Recife II. Recife-PE. Antonio Gonçalves de Oliveira; H. Nove de Julho. São Paulo-SP. Mariza D’Agostino Dias; H. Boa Viagem. Recife-PE. Antonio Gonçalves de Oliveira; H. Unimed - Franca. Franca-SP. Júlio C. B. Lucas; H. Clínicas da FMUSP. São Paulo-SP. Mariza D'Agostino Dias; Sociedade Hospitalar Samaritano. São Paulo-SP. Responsável não identificado; H. do Trabalhador. Curitiba-PR. Álvaro Réa Neto e Cristine de Oliveira; H. Barra D'Or. Rio de Janeiro-RJ. Juan Carlos Rosso Verdeal; H. Santa Joana. Recife-PE. Odin Barbosa da Silva; H. e Maternidade Ana Néri. Recife-PE. Antonio Gonçalves de Oliveira; H. Unimed. Campo Grande -MS. Sérgio Félix Pinto; H. NorteCor. Rio de Janeiro-RJ.Luiz Osório; H. Unimed Cuiabá. Cuiabá-M. Responsável não identificado; H. Univ. Sul Fluminense. Vassouras-RJ. Gerson Luiz de Macedo; H. ProntoCor Lagoa. Rio de Janeiro-RJ. Glaucia Moraes; H.Universitário C.Fraga
Filho-UFRJ. Rio de Janeiro-RJ. Frederico M. Salomão; Hospital Antônio Bezerra de Faria. Vila Velha-ES. José Roberto P. Santos; H. Santa Casa de Misericórdia de Maceió. Maceió-AL. Hélvio Chagas Ferro; Hospital São Lucas. Aracaju-SE. Celi Marques Santos; Hospital Guadalupe. Belém-PA. Leila Rezegue; Hospital de Acidentados/Clínica Santa Isabel. Goiânia-GO. Nara Elizia; H. Cardioeste. Rio de Janeiro-RJ. José Roberto P. Santos; H. São Francisco/Hosp Coração. Ribeirão Preto-SP. Marcus Antônio Ferez; Hospital Santa Casa de Porto Alegre. Porto Alegre-RGS. Gilberto Friedman e Jorge Hamilton Höher; H. e Maternidade São Camilo. São Paulo-SP. Edwin Koterba; H. São Camilo - Ipiranga. São Paulo-SP. Edwin Koterba.

\section{REFERÊNCIAS}

01. Martin GS, Mannino DM, Eaton S et al - Epidemiology of sepsis in the United States from 1979 through 2000. N Engl J Med, 2003;348:15461554.

02. Angus DC, Wax RS - Epidemiology of sepsis: an update. Crit Care Med, 2001;29;(Suppl7):S109-S116.

03. Friedman $\mathrm{G}$, Silva $\mathrm{E}$, Vincent $\mathrm{JL}$ - Has the mortality of septic shock changed with time? Crit Care Med, 1998;26:2078-2086.

04. Ananne D, Aegerter P, Jars-Guincestre MG et al - Current Epidemiology of Septic Shock: the CUB-Rea Network. Am J Resp Crit Care Med, 2003;168:165-172.

05. Angus DC, Linde-Zwirble WT, Lidicker $\mathrm{J}$ et al - Epidemiology of severe sepsis in the United States: analysis of incidence, outcome, and associated costs of care. Crit Care Med, 2001;29:1303-1310.

06. Van den Berghe $\mathrm{G}$, Wouters $\mathrm{P}$, Weekers $\mathrm{F}$ et al - Intensive insulin therapy in critically ill patients. N Engl J Med, 2001;345:1359-1367.

07. Annane D, Sébille V, Charpentier C et al - Effect of treatment with low doses of hydrocortisone and Fludrocortisone on mortality in patients with septic shock. JAMA, 2002;288:862-871.

08. Bernard GR, Vincent JL, Laterre PF et al - Efficacy and safety of recombinant human activated protein $\mathrm{C}$ for severe sepsis . N Engl J Med, 2001;344:699-709.

09. Vincent JL, Bernard GR, Beale R et al - Drotrecogin alfa (activated) treatment in severe sepsis from the global open-label trial ENHANCE: Further evidence for survival and safety and implications for early treatment. Crit Care Med, 2005;33:2266-2277.

10. Bone RC, Balk RA, Cerra FB, et al - Definitions for sepsis and organ failure and guidelines for the use of innovative therapies in sepsis. Chest, 1992:101:1644-1655.

11. Vincent JL, Moreno R, Takala J et al - The SOFA (Sepsis-related Organ Failure Assessment) score to describe organ dysfunction/failure. Intensive Care Med, 1996;22:707-710

12. Brun-Buisson C, Meshaka P, Pinton P et al - EPISEPSIS: a reappraisal of the epidemiology and outcome of severe sepsis in French intensive care units. Intensive Care Med, 2004;30:580-588

13. Padkin A, Goldfrad C, Brady AR, Young D, Black N, Rowan K - Epidemiology of severe sepsis occurring in the first $24 \mathrm{hrs}$ in intensive care units in England, Wales, and Northern Ireland. Crit Care Med, 2003;31:23322338.

14. Vincent JL, Sakr Y, Sprung CL et al - Sepsis in European intensive care units: Results of the SOAP study. Crit Care Med, 2006;34:344-353.

15. Silva E, Pedro Mde A, Sogayar AC et al - Brazilian Sepsis Epidemiological Study (BASES study). Crit Care, 2004;8:R251-R260.

16. Dellinger RP, Carlet JM, Masur H, Gerlach H, Calandra T, Cohen J, GeaBanacloche J, Keh D, Marshall JC, Parker MM, Ramsay G, Zimmerman JL, Vincent JL, Levy MM - Surviving Sepsis Campaign Management Guidelines Committee. Surviving Sepsis Campaign guidelines for management of severe sepsis and septic shock. Crit Care Med, 2004;32:21692170. 
17. Vincent JL - Dear SIRS, I'm sorry to say that I don't like you. Crit care Med, 1997;25:372-374.

18. Rangel-Frausto MS, Pittet D, Costigan M et al - The natural history of the systemic inflammatory response syndrome (SIRS). A prospective study. JAMA, 1995;273:117-123.

19. Brun-Buisson $\mathrm{C}$, Doyon $\mathrm{F}$, Carlet $\mathrm{J}$ et al - Incidence, risk factors, and outcome of severe sepsis and septic shock in adults. A multicenter prospective study in intensive care units. JAMA, 1995;274:968-974.

20. Sundararajan V, Maclsaac C, Presneill JJ, Cade JF, Visvanathan K - Epidemiology of sepsis in Victoria, Australia. Crit Care Med, 2005;33:71-80.

21. Alberti C, Brun-Buisson C, Burchardi $\mathrm{H}$ et al - Epidemiology of sepsis and infection in ICU patients from an international multicentre cohort study. Intensive Care Med, 2002;28:108-121.

22. Sands KE, Bates DW, Lanken PN et al - Epidemiology of sepsis syndrome in 08 academic medical centers. JAMA, 1997;278:234-240.

23. Baughman RP - Antibiotic resistance in the intensive care unit. Curr Opin Crit Care 2002, 8:430-434.

24. 24 .Clark NM, Patterson J, Lynch JP - Antimicrobial resistance among gram-negative organisms in the intensive care unit. Curr Opin Crit Care, 2003;9:413-423.

25. Clark NM, Hershberger E, Zervos MJ, Lynch JP - Antimicrobial resistance among gram-positive organisms in the intensive care unit. Curr Opin Crit Care, 2003;9:403-412.

26. Rivers E, Nguyen B, Havstad S et al - Early goal-directed therapy in the treatment of severe sepsis and septic shock. N Engl J Med, 2001;345:1368-1377.

27. Marik PE, Zaloga GP - Adrenal insufficiency during septic shock. Crit Care Med, 2003;31:141-145.

28. Bernard GR, Artigas A, Brigham KL et al - The American-European Consensus on ARDS. Definitions, mechanisms, relevant outcomes, and clinical trial coordination. Am J Respir Crit Care Med,1994;149:818-824.

29. Alberti C, Brun-Buisson C, Chevret $\mathrm{S}$ et al - Systemic inflammatory response and progression to severe sepsis in critically ill infected patients. Am J Respir Crit Care Med, 2005;171:461-468.

30. Abraham E, Matthay MA, Dinarello CA et al - Consensus conference definitions for sepsis, septic shock, acute lung injury, and acute respiratory dis- tress syndrome: time for a reevaluation. Crit Care Med, 2000;28:232-235.

31. Wenzel RP, Edmond MB - Severe sepsis - national estimates. Crit Care Med, 2001;29:1472-1474.

32. Pittet D, Rangel-Frausto S, Li N et al - Systemic inflammatory response syndrome, sepsis, severe sepsis and septic shock: incidence, morbidities and outcomes in surgical ICU patients. Intensive Care Med, 1995;21:302-309.

33. Wong MD, Shapiro MF, Boscardin WJ, Ettner SL - Contribution of major diseases to disparities in mortality. N Engl J Med, 2002;347:1585-1592.

34. Kieft H, Hoepelman Al, Zhou W, Rozenberg-Arska M, Struyvenberg A Verhoef $\mathrm{J}$ - The sepsis syndrome in a Dutch university hospital: clinical observations. Arch Intern Med, 1993;153:2241-2247.

35. Ferreira FL, Bota DP, Bross A et al - Serial evaluation of the SOFA score to predict outcome in critically ill patients. JAMA 2002;286:1754-1758.

36. Finfer S, Bellomo R, Lipman $\mathrm{J}$ et al - Adult-population incidence of severe sepsis in Australian and New Zealand intensive care units. Intensive Care Med, 2004;30:589-596.

37. Flaatten H - Epidemiology of sepsis in Norway in 1999. Crit Care, 2004;8: R180-R184.

38. Pinto GSS, David C, Japiassu AM et al - Glucose metabolism and tumor necrosis factor-alfa in gram-negative septic shock patients. Intensive Care Medicine, 2002;28:S194.

39. Vianna RC, David CM, Bozza FA et al - Antibiotic treatment in a murine model of sepsis: Impact on cytokines and endotoxin release. Shock, 2004;21:115-120.

40. Japiassú AM, Pinto JESS, David CM - Cinética do TNF-alfa em pacientes com choque séptico por bactérias gram negativas tratados com imunoglobulina policlonal humana $x$ placebo. Rev Bras Terap Intens, 2002;(Suppl):41-42.

41. David C - Sepse e Síndrome da Disfunção Orgânica Múltipla . Em: David C. Medicina Intensiva. Editora Revinter, Rio de Janeiro, 2004;563.

42. Levy, MM, Fink MP, Marshall JC et al - 2001 SCCM/ESICM/ACCP/ ATS/SIS International Sepsis Definitions Conference. Crit Care Med, 2003;31:1250-1256.

43. Peelen L, Keizer NF, Peek N et al - Influence of entry criteria on mortality risk and number of eligible patients in recent studies on severe sepsis. Crit Care Med, 2005;33:2178-2183. 